\title{
Multifocal metastases to choroid plexus from papillary thyroid carcinoma: illustrative case
}

\author{
Isidora R. Beach, BA, ${ }^{1}$ Adam M. Olszewski, MD, ${ }^{2}$ Alissa A. Thomas, MD, ${ }^{3}$ John C. DeWitt, MD, PhD, ${ }^{4}$ and Brandon D. Liebelt, $\mathrm{MD}^{2}$ \\ ${ }^{1}$ Larner College of Medicine, University of Vermont, Burlington, Vermont; and Departments of ${ }^{2}$ Neurosurgery, ${ }^{3}$ Neurological Sciences, and ${ }^{4}$ Pathology \& Laboratory Medicine, \\ University of Vermont Medical Center, Burlington, Vermont
}

\begin{abstract}
BACKGROUND Choroid plexus metastases are extremely rare from all types of malignancy, with only 42 cases reported in the literature thus far. Most of these originate from renal cell carcinoma and present as a solitary choroid plexus lesion; only two cases of multifocal choroid plexus metastases have been reported to date.

OBSERVATIONS The authors report the third case of multifocal metastases to the choroid plexus, that of a 75-year-old man who developed three measurable choroid plexus lesions approximately 3.5 years after undergoing total thyroidectomy and chemotherapy for papillary thyroid carcinoma. He underwent intraventricular biopsy of the largest lesion and subsequently died of hydrocephalus after opting for comfort care only.

LESSONS This is the third case of multifocal choroid plexus metastasis in the literature and the second case of multifocal metastasis from thyroid carcinoma. As such, the natural disease course is not well characterized. This case is compared with the previous eight reports of choroid plexus metastases from thyroid carcinoma, seven of which involved solitary lesions. The eight prior cases are evaluated with attention to treatment modalities used and factors potentially influencing prognosis, specifically those that might contribute to hydrocephalus, a reported complication for this pathology.
\end{abstract}

https://thejns.org/doi/abs/10.3171/CASE21436

KEYWORDS choroid plexus; metastases; hydrocephalus; neuroendoscopy

The choroid plexus (CP) is an uncommon location for metastases from central nervous system (CNS) and non-CNS malignancies, with only 42 cases reported in the literature. ${ }^{1-4}$ Eight cases of $\mathrm{CP}$ metastases from thyroid carcinoma have been reported previously, with only two confirmed cases of multifocal CP metastases known to date. ${ }^{1,5-11}$ All eight cases involved differentiated thyroid carcinoma (DTC), namely papillary or follicular subtypes. Only two known cases of multifocal metastasis to the CP from any malignancy exist in the literature, originating from papillary thyroid carcinoma and renal cell carcinoma, respectively. ${ }^{1,12}$

Brain metastasis from differentiated thyroid carcinoma suggests a poor prognosis. Differentiated thyroid carcinoma without brain metastasis has a $90 \%$ 10-year survival rate, whereas the presence of any brain metastases from DTC is associated with a $67 \%$ mortality rate and a median survival rate of 12.4 months from diagnosis. ${ }^{13,14}$ Review of nine cases of CP metastasis from DTC suggests that this entity likely bears an even poorer prognosis than that of overall brain metastases from DTC. Hydrocephalus was present in at least three of the nine cases-those with the worst outcomes, suggesting the presence of hydrocephalus as another poor prognostic indicator. We present the ninth case of CP metastasis from thyroid carcinoma, which is also unique in being the second of these presenting with multifocal $\mathrm{CP}$ lesions, a likely marker of poor prognosis. ${ }^{1}$

\section{Illustrative Case}

The patient was a 75-year-old man with a history of papillary thyroid carcinoma (PTC), tall cell variant, metastatic to the neck and lung, who presented with altered mental status, severe headache, low back pain, and urinary retention. He had been diagnosed with PTC 4 years prior and was treated with total thyroidectomy,

ABBREVIATIONS CNS = central nervous system; $\mathrm{CP}=$ choroid plexus; CSF = cerebrospinal fluid; $\mathrm{CT}=$ computed tomography; $\mathrm{DTC}=$ differentiated thyroid carcinoma; EVD = external ventricular drain; IVH = intraventricular hemorrhage; $M R I=$ magnetic resonance imaging; PTC = papillary thyroid carcinoma. INCLUDE WHEN CITING Published September 13, 2021; DOI: 10.3171/CASE21436

SUBMITTED July 29, 2021. ACCEPTED August 2, 2021.

(C) 2021 The authors, CC BY-NC-ND 4.0 (http://creativecommons.org/licenses/by-nc-nd/4.0/). 

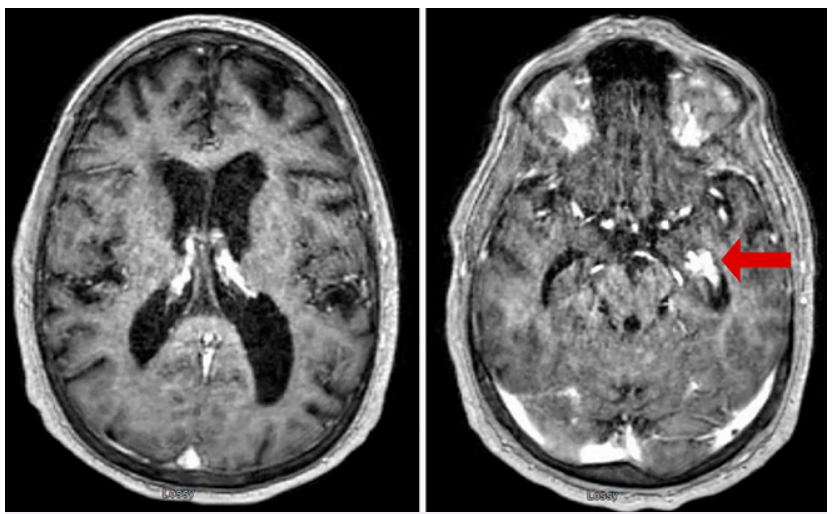

FIG. 1. Preoperative axial T1-weighted postcontrast MRI from initial presentation showing enhancing nodularity and thickening of the choroid plexus in the lateral ventricles (left) and nodule in the left lateral ventricle temporal horn (right, arrow).

cervical lymph node excision, and lenvatinib therapy. He later developed further metastasis to the C5 vertebral body and was treated with radiation and continued lenvatinib.

The patient had undergone brain magnetic resonance imaging (MRI) 1 month before presentation after seeing his primary oncologist for several days of headache, urinary frequency, and low back pain. This imaging revealed several $\mathrm{CP}$ abnormalities, including a $1.6 \times 0.9-\mathrm{cm}$ nodule in the temporal horn of the left lateral ventricle and avidly enhancing nodularity and thickening of $\mathrm{CP}$ in both
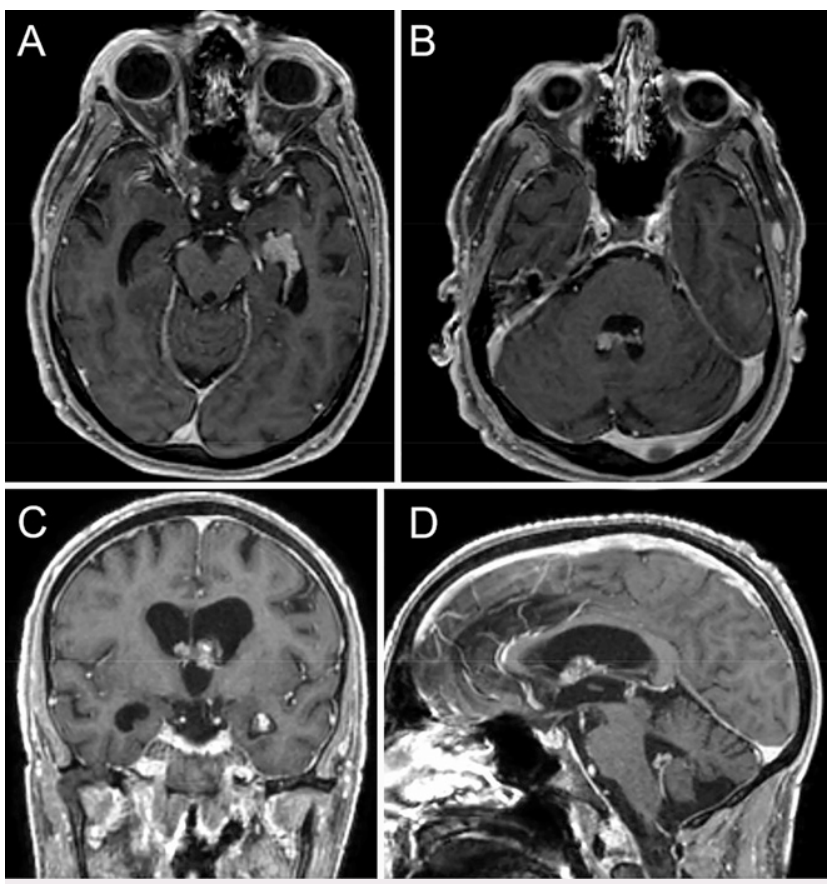

FIG. 2. Repeat preoperative T1-weighted postcontrast MRI showing progression of malignancy. A: Axial view showing interval increase in left temporal horn nodule. B: Axial view showing nodule in fourth ventricle. C: Coronal view showing enhancement, thickening, and nodularity of $\mathrm{CP}$ in both lateral ventricles (left greater than right), with rightward bowing of the septum pellucidum. D: Sagittal view showing $\mathrm{CP}$ nodule occluding the foramen of Monro.
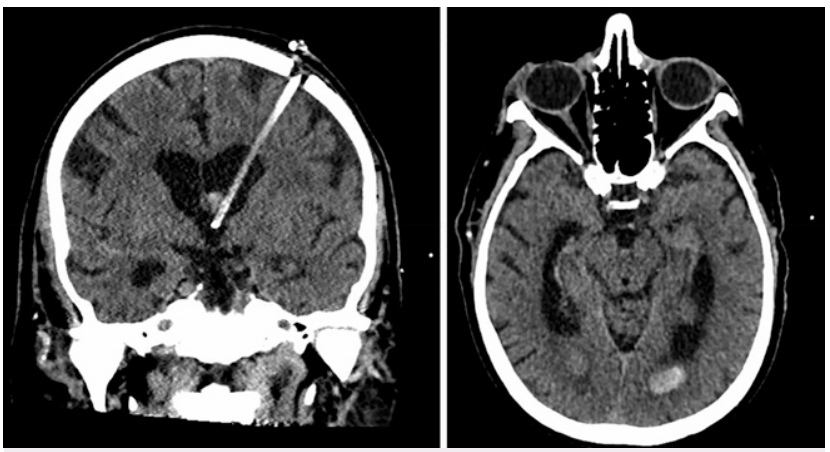

FIG. 3. Postoperative coronal CT shows postoperative changes from biopsy with EVD placement in left lateral ventricle (left) and persistent IVH in both lateral ventricles on axial view (right).

lateral ventricles and the fourth ventricle (Fig. 1). There were also trace dependent blood products in the occipital horns of both lateral ventricles and a slight rightward midline shift. These findings were concerning for intraventricular metastatic disease with mild associated intraventricular hemorrhage (IVH).

When the patient presented to the emergency department about 1 month later, his neurological examination was characterized by inattention, perseveration, and difficulty with multistep commands. He also had a postvoid residual of $578 \mathrm{~mL}$ and mild hyponatremia. MRI with contrast demonstrated numerous enhancing CP lesions in the body and temporal horn of the left lateral ventricle and nodular enhancement along the CP in the fourth ventricle (Fig. 2). A small enhancing lesion was seen in the third ventricle along the superior aspect of the cerebral aqueduct. The lateral and third ventricles had

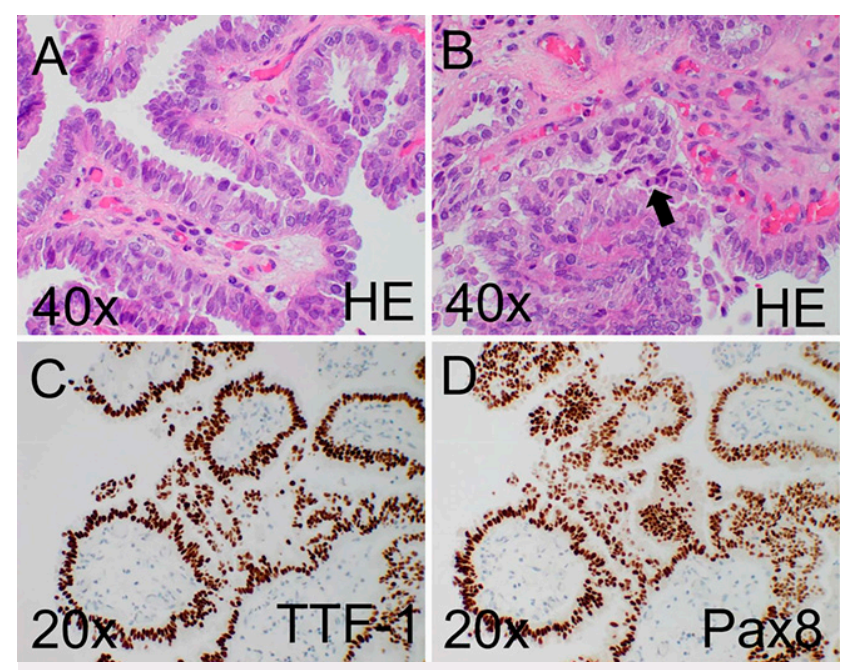

FIG. 4. Histology of choroid plexus metastasis. Microscopic examination shows an epithelial tumor with moderate pleomorphism growing in a papillary architecture with tall cell morphology (A and B) and scattered mitoses (arrow, B), consistent with a metastatic carcinoma. Immunohistochemical work-up showed tumor cells were positive for TTF-1 (C) and Pax8 (D), as well as CK7 (not shown), consistent with the patient's known primary papillary thyroid carcinoma, tall cell variant. $A$ and $B$ are shown at $\times 40$ magnification; $C$ and $D$ are shown at $\times 20$ magnification. $\mathrm{HE}=$ hematoxylin and eosin; TTF $=$ thyroid transcription factor. 


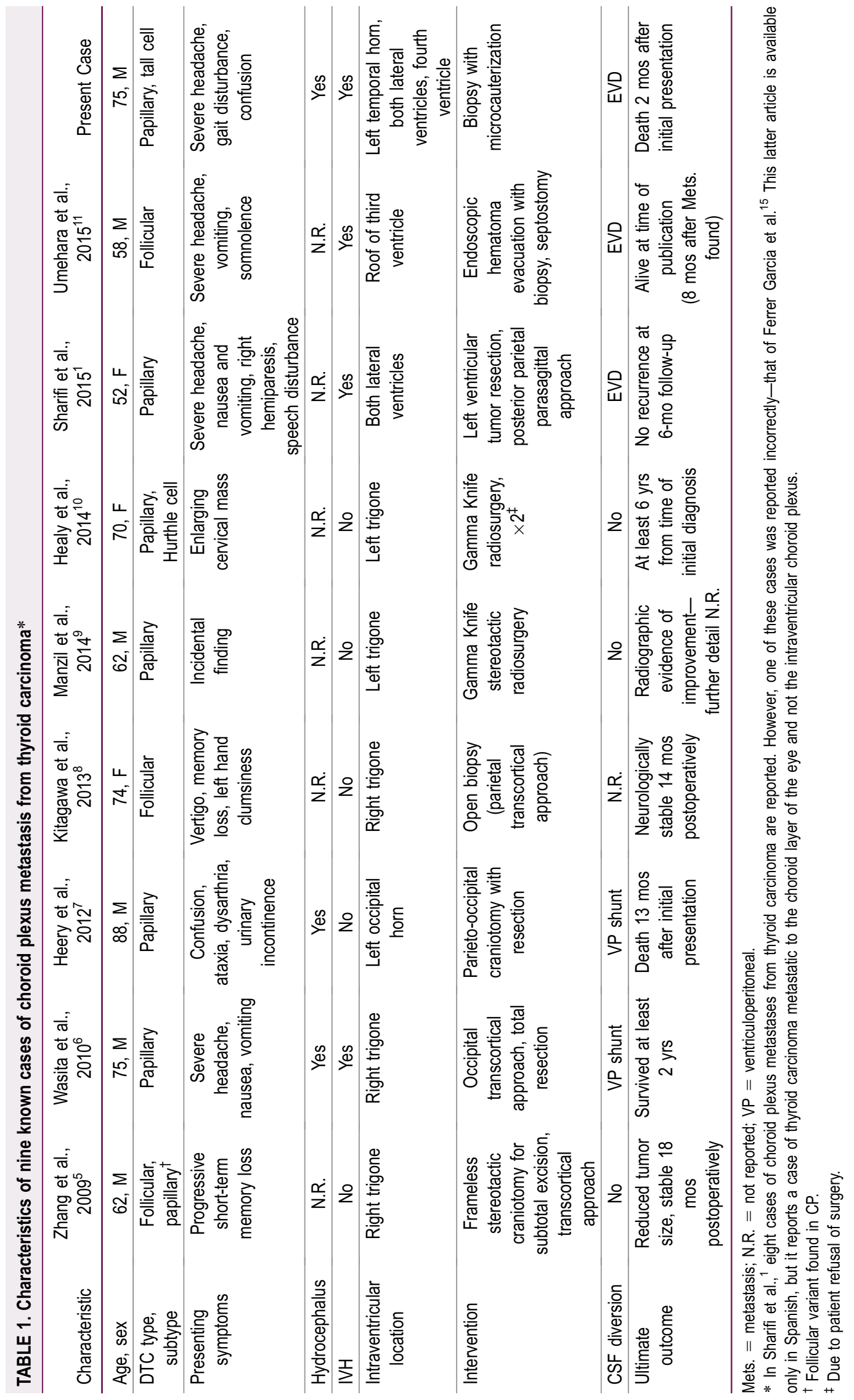


increased in size from the previous MRI with associated small IVH. Due to concern for metastatic disease and developing hydrocephalus, the patient underwent left frontal endoscopic tumor biopsy and placement of an external ventricular drain (EVD).

During the operation, a hemorrhagic-appearing mass was found nearly occluding the foramen of Monro (Fig. 2D). A left frontal endoscopic approach was chosen, the hemorrhagic mass was biopsied, and associated hematoma was removed, with the goal of removing the obstructive blood products to improve cerebrospinal fluid (CSF) drainage. The mass bled at the time of biopsy and was extensively cauterized, but visualization was partially obscured due to new and old blood products in the CSF. Underneath the hemorrhagic mass, the $\mathrm{CP}$ was abnormally hyperemic and enlarged. The left lateral ventricle was irrigated extensively to remove blood products, and an EVD was placed in the left lateral ventricle.

Follow-up computed tomography (CT) on postoperative day 1 revealed expected postoperative changes and decreased size of the CP lesion in the left lateral ventricle (Fig. 3). The surgical pathology returned as metastatic papillary thyroid cancer, tall cell variant, and molecular testing later demonstrated a v-raf murine sarcoma viral oncogene homolog B V600E mutation (Fig. 4). The patient's EVD was clamped with serial CT showing stable ventricular size until its removal on postoperative day 7 . His neurological status at first fluctuated between full orientation and confusion, but it soon progressively declined. A repeat head CT revealed interval increase in ventricular size with persistent IVH.

Continued urinary retention and back pain prompted a full spine MRI, which revealed numerous thoracic spine lesions, a new L1 vertebral body lesion, and an increased C5 vertebral body lesion. Given the severe burden of disease, the patient was transitioned to a comfort-directed plan of care on postoperative day 15 . He was discharged to a hospice and died about 1 week later.

\section{Discussion}

\section{Observations}

Metastatic disease to the CP is uncommon, with only 42 reports in the literature from any solid tumor primary malignancy. ${ }^{1-4}$ Thyroid carcinomas are rare as well, representing $1 \%$ of all malignancies. ${ }^{6}$ Thyroid carcinoma metastasizes to the brain in only $0.9 \%-1.5 \%$ of cases and has been reported in the $\mathrm{CP}$ in eight prior cases. ${ }^{1,5-11}$ Of the nine total cases, this is only the second involving multifocal CP lesions. ${ }^{1,5-11}$ Metastatic spread to the CNS in general is a sign of end-stage disease, but with limited reports of thyroid carcinoma metastatic to the $\mathrm{CP}$, prognostication for this diagnosis is difficult. This case is thus a rare presentation of a rare entity with a markedly poor outcome; the patient had a rapid decline and survived a little more than 2 months from the time CP metastases were diagnosed. We suspect that the hemorrhagic metastases leading to IVH and poor CSF reabsorption led to progressive hydrocephalus, increased intracranial pressure, and ultimately death.

\section{Lessons}

The aggressive disease course in this case supports the theorized mechanism for CP metastases that recognizes high tumor burden and favorable CNS entry (across the blood-brain barrier) as important factors in determining spread to this uncommon location. ${ }^{8}$ Our patient with a very high tumor burden developed multiple CP metastases, further suggesting that multifocal $\mathrm{CP}$ metastases reflect primary disease with a higher burden than that of disease presenting with a solitary metastatic lesion. Multifocality of lesions may thus be an indicator of poorer prognosis.

Table 1 summarizes key points from the eight previously reported cases of CP metastases from DTC to identify other potential prognostic indicators. ${ }^{1,5-11}$ Patients in the cases with the two worst outcomes had clinical and radiographic evidence of hydrocephalus, one of which was complicated by $\mathrm{IVH}^{6,7}$ Overall, IVH was present in four of the cases, with hydrocephalus present in at least three. CSF diversion procedures (EVD or ventriculoperitoneal shunt) were employed in at least five of the nine cases, suggesting the presence of hydrocephalus in more than three of these cases.

There is no treatment protocol for DTC metastases to CP due to the limited number of cases, but options have included resection, whole-brain radiation, Gamma Knife stereotactic radiosurgery, and radioiodine therapy (as an adjunctive therapy). ${ }^{1,5-11}$ Radiosurgery has not been used as a standalone approach in cases of CP metastasis from DTC; it has been used only as adjunctive therapy after surgical intervention. In all types of thyroid carcinoma presenting with solitary brain metastasis, there is a positive association between resection and increased survival time: 3-10 months, on average, with no surgical intervention compared with 17-54 months after resection. ${ }^{5}$ The one known reported case of multifocal brain metastasis from thyroid carcinoma was also treated with resection, but the outcome after 6 months was not reported. ${ }^{1}$ Surgery may be complicated by IVH due to the hyperemic nature of CP metastases, which neurosurgeons should be prepared to manage intraoperatively using irrigation, cautery, and CSF diversion procedures. Hydrocephalus, a likely factor in the majority of these cases, can be worsened by IVH, a complication that should be anticipated.

\section{Conclusions}

Thyroid carcinoma metastatic to the $\mathrm{CP}$ is exceedingly rare and typically presents as a solitary lesion. This is the second known reported case of multifocal $\mathrm{CP}$ metastasis from thyroid carcinoma specifically. ${ }^{1}$ Multifocal CP metastases are likely the manifestation of a more aggressive malignancy; if there is suspicion for the diagnosis, an endoscopic intraventricular biopsy with EVD placement may be performed to confirm the diagnosis. Resection can be considered for solitary and obstructive lesions, but because the entity is rare, further evidence is necessary to make stronger recommendations. This case expands the limited documentation of metastatic thyroid carcinoma to the choroid plexus and, along with previous reports, suggests an association with hydrocephalus that should be managed by CSF diversion.

\section{Acknowledgments}

We thank Dr. Paul Unger, the primary oncologist, for his care of this patient.

\section{References}

1. Sharifi G, Bakhtevari MH, Alghasi M, Nosari MA, Rahmanzade R, Rezaei O. Bilateral choroid plexus metastasis from papillary thyroid carcinoma: case report and review of the literature. World Neurosurg. 2015;84(4):1142-1146.

2. Sellier A, Russo D, Robertson FC, et al. A choroid plexus metastasis of a prostatic adenocarcinoma mimicking a choroid plexus carcinoma: a case report. Neurochirurgie. Published online March 3, 2021. doi: 10.1016/j.neuchi.2021.02.011.

3. Amirjamshidi A, Abbasioun K, Ghassemi B, Hamidi M. Solitary metastasis of adenocarcinoma of submandibular gland to choroid 
plexus in cerebellopontine angle: first case reported in literature. World Neurosurg. 2019;128:162-164.

4. Shenoy S, Shenoy SN. Progeny in an inhospitable milieu-solitary intraventricular metastasis from a triple-negative breast cancer mimicking central neurocytoma: case report and review of diagnostic pitfalls and management strategies. World Neurosurg. 2020;135: 309-315.

5. Zhang YA, Kavar B, Drummond KJ. Thyroid carcinoma metastasis to the choroid plexus of the lateral ventricle. $J$ Clin Neurosci. 2009;16(1):118-121.

6. Wasita B, Sakamoto M, Mizushima M, Kurosaki M, Watanabe T. Choroid plexus metastasis from papillary thyroid carcinoma presenting with intraventricular hemorrhage: case report. Neurosurgery. 2010;66(6):E1213-E1214.

7. Heery CR, Engelhard HH, Slavin KV, Michals EA, Villano JL. Unusual CNS presentation of thyroid cancer. Clin Neurol Neurosurg. 2012;114(7):1107-1109.

8. Kitagawa Y, Higuchi F, Abe Y, Matsuda H, Kim P, Ueki K. Metastasis to the choroid plexus from thyroid cancer: case report. Neurol Med Chir (Tokyo). 2013;53(11):832-836.

9. Manzil FF, Bender LW, Scott JW. Evaluation of rare choroid plexus metastasis from papillary thyroid carcinoma with multimodality imaging. Clin Nucl Med. 2014;39(6):551-553.

10. Healy AT, Otvos B, Schroeder J, Hamrahian AH, Angelov L, Kamian K. Hurthle cell carcinoma presenting as a single choroid plexus metastasis. J Clin Neurosci. 2014;21(8):1448-1450.

11. Umehara T, Okita $Y$, Nonaka M, et al. Choroid plexus metastasis of follicular thyroid carcinoma diagnosed due to intraventricular hemorrhage. Intern Med. 2015;54(10):1297-1302.

12. Hillard VH, Musunuru K, Hasan I, Zia S, Hirschfeld A. Long-term management of bilateral metastases of renal cell carcinoma to the choroid plexus. Acta Neurochir (Wien). 2003;145(9):793-797.
13. Chiu AC, Delpassand ES, Sherman SI. Prognosis and treatment of brain metastases in thyroid carcinoma. J Clin Endocrinol Metab. 1997;82(11):3637-3642.

14. Liao S, Shindo M. Management of well-differentiated thyroid cancer. Otolaryngol Clin North Am. 2012;45(5):1163-1179.

15. Ferrer García JC, Merino Torres JF, Ponce Marco JL, Piñón Sellés F. Unusual metastasis of differentiated thyroid carcinoma. Article in Spanish. Med Interna. 2002;19(11):579-582.

\section{Disclosures}

The authors report no conflict of interest concerning the materials or methods used in this study or the findings specified in this paper.

\section{Author Contributions}

Conception and design: Liebelt, Beach, Olszewski. Acquisition of data Beach, Thomas, DeWitt. Analysis and interpretation of data: Beach, Thomas. Drafting the article: Liebelt, Beach, DeWitt. Critically revising the article: all authors. Reviewed submitted version of manuscript: Liebelt, Beach, Olszewski, Thomas. Approved the final version of the manuscript on behalf of all authors: Liebelt. Administrative/technical/ material support: Beach, Olszewski. Study supervision: Liebelt, Beach, Olszewski.

\section{Correspondence}

Brandon D. Liebelt: University of Vermont Medical Center, Burlington, VT. brandon.liebelt@uvmhealth.org. 